\title{
From Web Requirements to Navigational Design - A Transformational Approach
}

\author{
Pedro Valderas, Joan Fons, and Vicente Pelechano \\ Department of Information System and Computation \\ 46022, Technical University of Valencia, Spain \\ \{pvalderas,jjfons,pele\}@dsic.upv.es
}

\begin{abstract}
Although MDA defines each stage for building software from models, it does not specify any concrete technique. In this sense, in the Web applications development, little methodological support is provided to both define and apply model to model transformations. In this work, we present a strategy based on graph transformations in order to define and to automatically apply model to model transformations. This strategy has been used to automate the OOWS CIM to PIM transformation.
\end{abstract}

\section{Introduction}

In the MDA [5] development process a computational independent model (CIM), a platform independent model (PIM) and a platform specific model (PSM) are proposed in order to describe the system at different levels of abstraction. Besides the specification of these models, MDA proposes that a set of consecutive transformations should be applied in order to transform these models into code. However, in the Web applications development area, little methodological support is provided to both define and apply model to model transformations

In this work, we introduce a strategy that is based on graph transformations in order to define and to automatically apply model to model transformations. This strategy has been used to automate the OOWS [2] CIM to PIM transformation. This contribution allows us to provide a fully MDA approach that support automatic model transformation.

This paper is organized as follows: Section 2 presents the OOWS method. Section 3 proposes a strategy to automate the CIM to PIM transformation. Finally, conclusions and future work are presented in section 4 .

\section{OOWS: A MDA-Based Method for the Web Applications Development}

The OOWS [2] development process begins by describıng the early requirements of a Web application in the CIM model. Next, the PIM model is obtained by applying a model-to-model transformation. This model describes the Web application with highlevel constructs that hide the necessary details for a particular platform. Finally, an automatic transformation is applied to obtain code from the PIM model.

This work focuses on the CIM to PIM transformation. Then, to better understand this transformation, a brief overview of the OOWS CIM and PIM models is next presented. 


\subsection{The CIM Model}

The OOWS CIM model is defined from: (1) the identification of the tasks that users must achieve and (2) the description of these tasks from the system-user interaction.

\subsubsection{Task Identification}

To identify tasks, we propose the construction of a task taxonomy. To do this we take a statement of purpose that describes the goal for which the application is being built, as the starting point. The statement of purpose is considered as the most general task. Then, this task is refined into more specific ones until a group of elementary tasks are obtained. An elementary task is defined as a task that when divided into subtasks involves either the user or the system, but not both. In addition, we propose to enrich this taxonomy by indicating temporal relationships among tasks. To do this, we have used the relationships introduced by the CTT approach (ConcurTaskTree) [6].

Figure 1 shows a partial view of the task taxonomy that we obtain from the statement of purpose of a Web application for the sale of audiovisual and bibliographic products (CDs, DVDs and Books). In order to easily identify the elementary tasks they are circled with a thicker line.

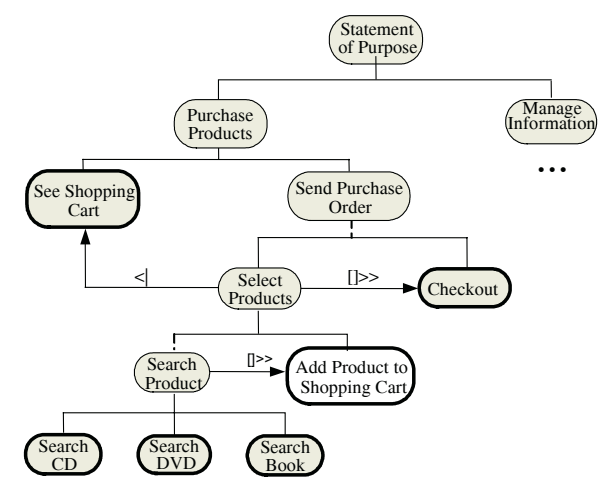

Fig. 1. A Task Taxonomy of an On-Line Sale Web application

\subsubsection{Task Description - Activity Diagrams and Interaction Points}

To describe elementary tasks we extend traditional description where user and system actions are described by indicating explicitly when (at which exact moment) the interaction between user and system is performed. We introduce the concept of interaction point (IP). In each IP, the system provides the user with information. Moreover, access to operations can also be provided. In this sense, the user can perform several actions with both the information and the operations: he/she can select information (as a result the system provides the user with new information) and he/she can activate an operation (as a result the system carries out an action).

To perform descriptions of this kind, we propose a graphical notation based on UML activity diagrams [1] (see Figure 2). Each node (activity) represents an IP (solid line) or a system action (dashed line). Finally, each arc represents (1) a user action (selection of information or operations) if the arc source is an IP or (2) a node sequence if the arc source is a system action. 
Figure 2 shows the description of the Search $C D$ elementary task. This task starts with an IP where the system provides the user with a list of music categories (1). Next, the user selects a category (2). Next, the task continues with an IP where the system informs about the CDs of the selected category (3). Depending on the user action there are two ways (A and B) to continue with the task: A) The user selects a CD (4a) and the task ends with an IP where the system provides the user with a description of the selected CD (5a). B) The user selects a search operation (4b). Then, the system performs a system action which searches the CDs of an artist (5b). Finally, since the search result is a list of CDS it is shown in the IP where the full list of CDs is previously shown $(6 \mathrm{~b})$.

As we can see, details about the information exchanged between the user and the system are not described. To do this, we propose a technique based on information templates such as the CRC Card [9].

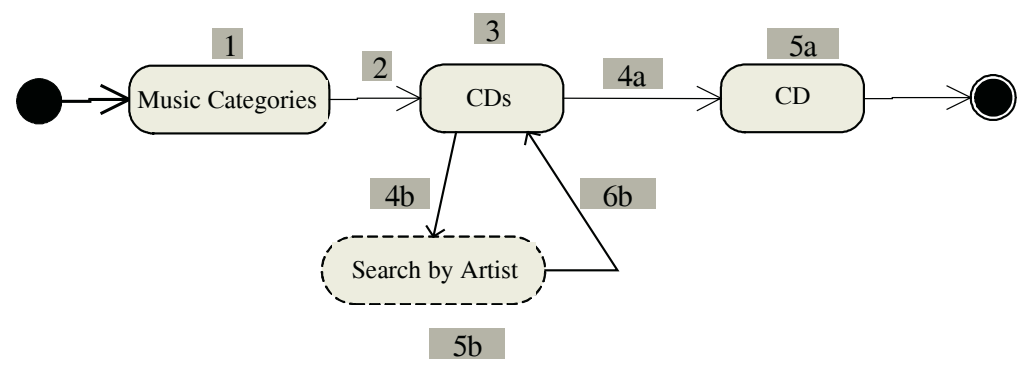

Fig. 2. Search CD Elementary Task

\subsection{The PIM Model}

The OOWS PIM model describes the different aspects of a Web application at a high level of abstraction. The system static structure and the system behaviour are described in three models (class diagram and dynamic-and functional models) that are borrowed from an object oriented software production method called OO-Method [7]. The navigational aspects of a Web application are described in a navigational model.

The navigational model [2] is represented by a directed graph (which defines the navigational structure) whose nodes are navigational contexts and its arcs denote navigational links. A navigational context (represented by an UML package stereotyped with the «context» keyword) defines a view on the class diagram that allows us to specify an information recovery. A navigational link represents navigational context reachability: the user can access a navigational context from a different one if a navigational link between both has been defined.

The navigational context is made up of a set of navigational classes that represent class views over the classes of the class diagram (including attributes and operations). Each navigational context has one mandatory navigational class, called manager class and optional navigational classes to provide complementary information of the manager class, called complementary classes. All navigational classes must be related by unidirectional binary relationships, called navigational relationships that are defined upon an existent relationship in the class diagram. 


\section{From a Task Description to the OOWS Navigational Model: Automating the CIM to PIM Transformation}

In this section, we present a strategy based on graph transformations that allow us to automatically derivate the OOWS PIM navigational model from the CIM model. This strategy is divided into two main stages: (1) Definition of the mapping rules that transform a model into another model and (2) Application of the mapping rules.

\subsection{Graph Transformations - Defining the Mapping Rules}

In order to define the mapping rules we have chosen a technique based on graph transformations. Graph transformations are specified using transformation systems. Transformation systems rely on the theory of graph grammars [8]. A transformation system is composed of several transformation rules. Technically, a rule is a graph rewriting rule equipped with negative application conditions and attribute conditions. Every rule is composed by a Left Hand Side (LHS), that defines a pattern to be matched in the source graph and a Right Hand Side (RHS) that defines the replacement for the matched subgraph if a Negative Application Condition (NAC) does not matches.

NAC LHS RHS

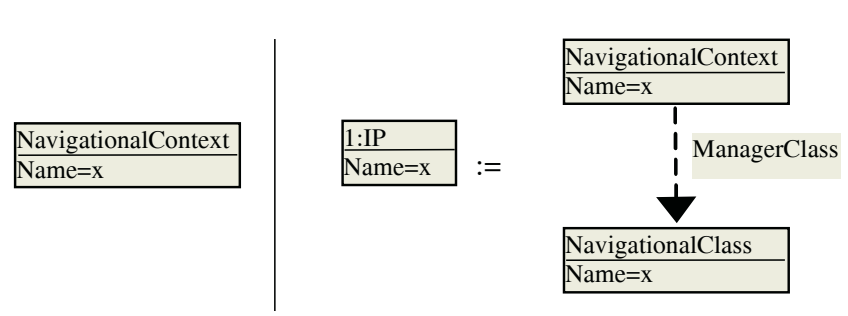

Fig. 3. A Transformation Rule

To define the OOWS CIM to PIM transformation we have defined a set of transformation rules. These rules transform a graph that represents a task description into a graph that represents an OOWS navigational model. Due to space constraints, we only present a representative rule that can be seen in Figure 3 . The rest of rules are identified in [3]. The rule of Figure 3 says that when an IP (LHS) is found it must be transformed into a Navigational Context with its Manager Navigational Class (RHS). However, this rule is not applied if the navigational context is already defined (NAC).

\subsection{Automatically Applying Graph Transformations}

To automatically apply graph transformations we propose the use of the Attribute Graph Grammar (AGG) tool [4]. AGG was chosen because it allows the graphical expression of directed, typed and attributed graphs for expressing rules. It has a powerful library containing notably algorithms for graph transformation, critical pair analysis, consistency checking and the application of positive and negative conditions. 
The AGG tool allows us to automatically transform a source graph into a target graph. However, models are not always defined as graphs. In these cases, to fully automate the model-to-model transformation by means of the AGG tool, two additional steps must be fulfilled: (1) The source model must be represented as a correct AGG graph and (2) once the graph transformations are applied into this graph, the obtained graph must be translated into the correct target modelling language.

Next, we present the strategy to achieve these steps in order to automate the OOWS CIM to PIM transformation.

\subsubsection{Obtaining an AGG Graph from a Task Description}

To automate the transformation of a task description into an AGG graph we propose a strategy based on the translation of XML documents. On one hand, the CIM model is specified in a XML document. This XML specification is built by means of XML elements defined from the task elements presented in section 2.1. On the other hand, the AGG system also uses XML to store its graphs by means of four XML elements: the NodeType and EdgeType elements that allow us to specify which kind of nodes and edges can be defined in the graph; and the Node and Edge elements that allow us to define the graph. Then, a translation of XML documents can be easily performed by a XSL Transformation.

The XSL transformation is performed by following the following three steps: (1) We transform the task taxonomy into an AGG graph (tasks are transformed into graph nodes and task relationships define graph edges). (2) Each activity diagram is represented as an AGG graph (IPs and System Actions are transformed into graph nodes while the activity diagrams arcs define graph edges). (3) The defined AGG graphs are joined into a single graph (each node that represents an elementary task is connected to the node that represents the initial IP or System Action of the activity diagram that describe the task).

\subsubsection{Obtaining an OOWS Specification from an AGG Graph}

When the AGG system transformation is finished we obtain a graph that represents an OOWS navigational model. This graph is made up of a set of nodes and edges defined from the OOWS metamodel elements. These elements have been briefly explained in section 3.2. Figure 4 shows a partial view of the graph that represents the OOWS navigational model of the "Web Sale application". This graph is obtained after applying the full set of transformation rules into the CIM model. According to this figure, two navigational contexts are defined: the $C D$ navigational context and the Cart navigational context. On one hand, the $C D$ navigational context is made up of a manager class (with some attributes and an operation) and a complementary class (with an attribute). On the other hand, the Cart navigational context is made up of the manager class with an attribute and an operation (with its parameters). In addition, a link is defined between both contexts.

Following a similar strategy than the previous one, an OOWS specification is obtained from an AGG graph by means of a translation of XML documents. On one hand, the AGG system stores the graph in a XML document. On the other hand, our OOWS case tool stores the OOWS specifications in XML repositories. The transformation between the two XML documents is performed by a XSL transformation. 


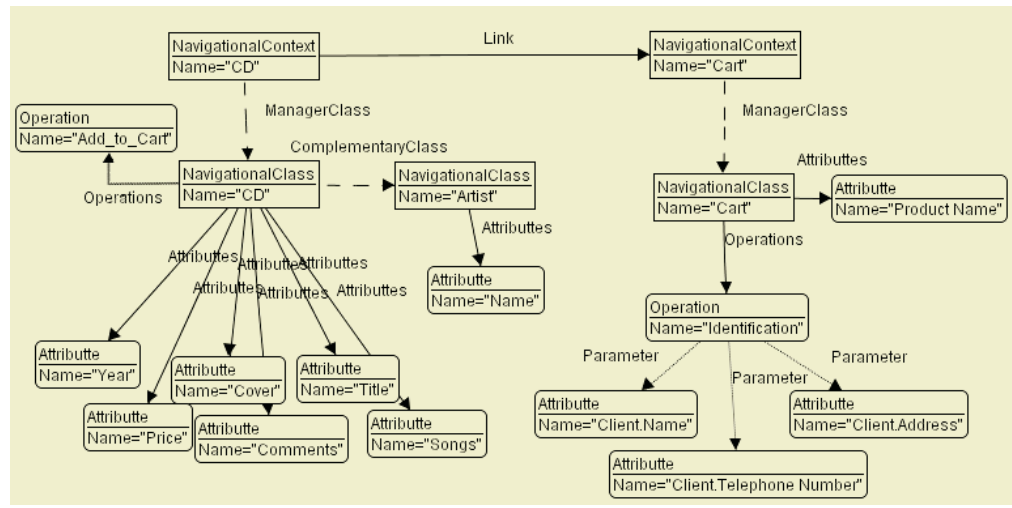

Fig. 4. An OOWS navigational model represented by means of an AGG graph

\section{Conclusions and Further Work}

We have present a strategy based on graph transformations that allow us to automate model to model transformations following the MDA approach. This strategy has been used to automate the CIM to PIM transformation of the OOWS method. To do this, the CIM and PIM models are represented as graphs by using XML translation based technique. Next, the graph transformations are applied by means of the AGG tool.

\section{References}

1. Object Management Group. Unified Modeling Language (UML) Specification Version 2.0 Final Adopted Specification. www.omg.org, 2003.

2. Fons, J., Pelechano, V., Albert, M., and Pastor, O.: Development of Web Applications from Web Enhanced Conceptual Schemas. In ER'03, volume 2813 of LNCS, 2003.

3. Valderas, P.: Capturing Web Application Requirements. Technical report, DSIC, Technical University of Valencia, February 2005. http://oomethod.dsic.upv.es.

4. The Attributed Graph Grammar System v1.2.4. http://tfs.cs.tu-berlin.de/agg/. 2004

5. Object Management Group. Model Driven Architecture (MDA). www.omg.org/mda, 2004.

6. Paternò, F., Mancini, C. and Meniconi, S.: "ConcurTaskTrees: a Diagrammatic Notation for Specifying Task Models", INTERACT'97, Chapman \& Hall, 362-369.

7. Pastor, O., Gomez, J., Insfran, E. and Pelechado V.: The OO-Method Approach for Information Systems Modelling: From Object-Oriented Conceptual Modeling to Automated Programming. Information Systems 26 (2001) 507-534

8. Rozenberg, G. (ed.): Handbook of Graph Grammars and Computing by Graph Transformation. World Scientific, Singapore (1997)

9. Wirfs-Brock, Wilkerson, B. and Wiener L.: Designing Object-Oriented Software. PrenticeHall, 1990. 